\title{
Immunochemical Localization of Keratan Sulfate Proteoglycans in Cornea, Sclera, and Limbus Using a Keratanase-Generated Neoepitope Monoclonal Antibody
}

\author{
Saeed Akbtar, ${ }^{1,2,3}$ Briedgeen C. Kerr, ${ }^{3,4}$ Anthony J. Hayes, ${ }^{4}$ Clare E. Hughes, ${ }^{4}$ \\ Keith M. Meek, ${ }^{1}$ and Bruce Caterson ${ }^{4}$
}

Purpose. To evaluate the use of neoepitope monoclonal antibody BKS-1, which recognizes keratanase-generated keratan sulfate (KS) stubs on keratan sulfate proteoglycans in human cornea, limbus, and sclera.

Methods. BKS-1 specifically recognizes a keratanase-generated neoepitope [ $N$-acetyl-glucosamine-6-sulfate (GlcNAc-6-S)] at the nonreducing terminal of corneal and skeletal KS glycosaminoglycan chains. It was produced by using keratanase-digested KS peptides from bovine cartilage aggrecan as the immunizing antigen. BKS-1 was used in conjunction with 5D4 to analyze the KS distribution in human cornea, limbus, and sclera using Western blotting, immunohistochemistry, and electron microscopy.

Results. 5D4 Western blot analysis displayed a diffuse staining pattern, and it was difficult to distinguish differences among cornea, sclera, and limbus. However, BKS-1 showed differences in KS levels, with higher levels in the cornea and lower levels in the limbus and sclera. Ultrastructural studies showed that the monoclonal antibody (mAb) BKS-1 neoepitope was not observed in the epithelium or basement membrane; however, 5D4 was present in these layers. Large quantities of both antibodies were present in Bowman's layer, stroma, and Descemet's membrane, but the quantity of 5D4 was significantly higher $(P<0.001)$ than the quantity of BKS-1 in all these layers of the cornea.

Concuusions. mAb 5D4 recognizes oversulfated structures within KS chains, whereas BKS-1 recognizes a single neoepitope on KS after keratanase digestion of monosulfated KS disaccharides. With the use of BKS-1, the authors identified a more clearly defined pattern for KS distribution in the cornea than was seen with 5D4. The presence of a large quantity of BKS-1 immunostaining in the cornea suggests that KS-substituted proteoglycans are more prevalent in the cornea than in

From the Schools of ${ }^{1}$ Optometry and Vision Sciences and ${ }^{4}$ Biosciences, Cardiff University, Cardiff, United Kingdom; and the ${ }^{2}$ Department of Optometry and Vision Sciences, College of Applied Medical Sciences, King Saud University, Riyadh, Saudi Arabia.

${ }^{3}$ These authors contributed equally to the work presented here and should therefore be regarded as equivalent authors.

Supported by Medical Research Council Program Grant G0001033 (KMM, BC) and the Arthritis Research Campaign, UK (BC). KMM is a Royal Society-Wolfson Research Merit Award holder.

Submitted for publication December 18, 2006; revised June 28 and November 14, 2007; accepted March 24, 2008.

Disclosure: S. Akhtar, None; B.C. Kerr, None; A.J. Hayes, None; C.E. Hughes, None; K.M. Meek, None; B. Caterson, None

The publication costs of this article were defrayed in part by page charge payment. This article must therefore be marked "advertisement" in accordance with 18 U.S.C. $\$ 1734$ solely to indicate this fact.

Corresponding author: Saeed Akhtar, Department of Optometry and Vision Sciences, College of Applied Medical Sciences, King Saud University, PO Box 1019, Riyadh 11433, Saudi Arabia;

akhtars@ksu.edu.sa. the limbus or sclera. (Invest Opbthalmol Vis Sci. 2008;49: 2424-2431) DOI:10.1167/iovs.06-1498

$\mathrm{C}$ orneal proteoglycans (PGs) are thought to play important roles in collagen fibrillogenesis and matrix assembly and in modifying the structure and function of collagen fibrils. ${ }^{1-3}$ They are macromolecules composed of a protein core with covalently linked glycosaminoglycan side chains. ${ }^{4}$ Studies from bovine, ${ }^{5}$ rabbit, ${ }^{6}$ chicken, ${ }^{7}$ monkey, ${ }^{8}$ and human ${ }^{9}$ corneas show that the predominant components of the glycosaminoglycans are keratan sulfate and dermatan sulfate, with smaller amounts of heparan sulfate. Experiments suggest that the core proteins on the keratan sulfate proteoglycan (KS-PG) act in conjunction with type $\mathrm{V}$ collagen to regulate the diameters of the collagen fibrils, ${ }^{10}$ whereas the sulfated gylcosaminoglycans appear to regulate fibril spacing. ${ }^{11} \mathrm{KS}$ is associated with positive staining bands $a$ and $c^{12}$ along the fibril. Dermatan sulfate may also be important for interfibrillar spacing and lamellar adhesion of collagen ${ }^{13}$ and is associated with bands $d$ and $e .^{12}$

Keratan sulfate proteoglycan (KS-PG), the most abundant carbohydrate in the cornea, plays an important role in the maintenance of corneal transparency. ${ }^{14,15}$ Three proteins, lumican, keratocan, and mimican, have been reported to carry keratan sulfate through $\mathrm{N}$-linked oligosaccharides in the cornea. ${ }^{16-19}$ The expression of these carrier proteins is regulated during eye development, suggesting the importance of KS-PG in corneal transparency. ${ }^{15,17-21}$

Several investigators have previously examined the structure and biosynthesis of KS. ${ }^{15,22-25}$ The general structure of KS is based on repeating disaccharides of $\mathrm{N}$-acetyl-lactosamine (-3Gal $\beta 1$ to 4 GlcNAc $\beta 1-$ ) in which most of the $N$-acetyl-glucosamine (GlcNAc) residues and some of the galactose (Gal) residues are sulfated on the C-6 position. ${ }^{26}$ Analysis of KS isolated from porcine and calf cornea revealed a domain structure in which the linkage region is followed by one or two unsulfated $\mathrm{N}$-acetyl-lactosamine disaccharides, a constant number of monosulfated $\mathrm{N}$-acetyl-lactosamine disaccharides followed by anything from 6 to 36 disaccharides of disulfated $\mathrm{N}$-acetyl-lactosamine residues. ${ }^{27}$ Monoclonal antibodies (mAbs) such as $5 \mathrm{D} 4$, raised in mice against skeletal KS from humans, ${ }^{28}$ have been helpful for a detailed analysis of the distribution of KS in cornea. The antibody recognizes linear disulfated $N$ acetyl-lactosamine disaccharides containing segments in KS. ${ }^{29-32}$ This antibody does not allow for the discrimination of different KS types (i.e., type 1 corneal KS vs. type 2 skeletal KS). Although it is useful for the detection of oversulfated KS chains, it is not suitable for selective quantification of tissuederived KS or diseased-derived KS. The new mAb BKS-1 recognizes a neoepitope containing the $N$-acetylglucosamine-6sulfate at the nonreducing terminal of KS GAG chains predigested with keratanase. ${ }^{33}$

In the present study, we used the new KS-stub mAb BKS-1 specifically for keratanase-generated neoepitopes in KS-PGs and compared it with the existing $\mathrm{KS}$ mAb 5D4 to investigate 
KS sulfation pattern motifs in normal corneal, limbal, and scleral PGs.

\section{Methods}

Tissue procurement and use were in accordance with the Declaration of Helsinki and local regulations. The study was approved by the local ethics committee, the Pontypridd \& Rhondda NHS Trust Ethical Committee. Healthy donor eyes were obtained from the US National Disease Research Interchange.

\section{Preparation and Screening of the Antigen}

The monoclonal antibody BKS-1, which recognizes keratanase neoepitope KS stubs after the keratanase digestion of KS-PGs, was produced $^{28}$ using KS peptides obtained from bovine nasal cartilage aggrecan as the immunizing antigen. ${ }^{34}$ The KS peptides were produced after trypsin digestion of bovine aggrecan, followed by pretreatment with keratanase before further purification by size-exclusion and ion-exchange chromatography. Screening for mAbs that recognize the keratanase-generated KS stubs involved ELISA plates coated with keratanase-digested KS-PGs from bovine cartilage (skeletal KS) and cornea (corneal KS). Immunopositive reactions were detected with skeletal and corneal KS-PGs after keratanase pretreatment; however, negative immunoreactivity for KS stubs was detected with positive recognition of only skeletal KS or reactivity with undigested KS-PGs. After secondary screening using Western blot analysis against purified corneal KS-PGs (keratocan and lumican predigested with keratanase vs. keratanase II, which acted as positive and negative controls, respectively), the mAb BKS-1 was identified as an IgM subclass with kappa light chain.

\section{Western Blot Analysis}

Healthy human (donor age, 24-54 years) cornea, sclera, and limbus were minced separately, and PGs were extracted twice for 24 hours each in $10(\mathrm{wt} / \mathrm{vol}) 4 \mathrm{M}$ guanidine $\mathrm{HCl}$ containing protease inhibitors ( $0.1 \mathrm{M}$ 6-amino hexanoic acid, $5 \mathrm{mM}$ benzamidine, $1 \mathrm{mM}$ phenylmethylsulfonyl fluoride, $10 \mathrm{mM}$ EDTA) in $0.05 \mathrm{M}$ sodium acetate, $\mathrm{pH} 6.8$. Pooled guanidine extracts were dialyzed against $6 \mathrm{M}$ urea, $0.1 \mathrm{M}$ Tris, $0.1 \%$ Chaps, $\mathrm{pH} 6.8$, at $4^{\circ} \mathrm{C}$. Anion exchange chromatography was carried out on both samples with bound PGs eluted in $2 \mathrm{M} \mathrm{NaCl}$ in $6 \mathrm{M}$ urea buffer, as described. Bound fractions were dialyzed to water, lyophilized, and reconstituted in $200 \mu \mathrm{L}$ water. Dimethylmethylene blue analysis was carried out to determine the sulfated glycosaminoglycan (GAG) content of the samples. Volumes equivalent to $2 \mu \mathrm{g} \mathrm{GAG}$ were left undigested for 5D4 analysis and keratanase (Seikagaku, Tokyo, Japan) with the use of $0.001 \mathrm{U}$ keratanase $/ 1 \mu \mathrm{g}$ GAG in $0.1 \mathrm{M}$ Tris-HCl, pH 6.8, for BKS-1 analysis. SDS-PAGE and Western blot analysis was carried on $4 \%$ to $20 \%$ Tris-glycine gradient gels using mAbs 5D4 and BKS-1 diluted 1:100 from neat hybridoma supernatant (approximately $0.1 \mu \mathrm{g} / \mathrm{mL}$ ).

With the use of corneal PG extracts, three experimental protocols were conducted. First, using the undigested PG extracts from the cornea, SDS-PAGE was performed, and transfers for Western blot analysis for two lanes of each sample were undertaken. One lane was immunostained with 5D4 and the other one with BKS-1. Second, the corneal extracts were predigested with keratanase before SDS-PAGE (two lanes) and transferred for Western blot analyses using mAbs 5D4 and BKS-1 on separate lanes. Third, corneal extracts were run on SDS-PAGE and transferred to nitrocellulose, where they were subjected to keratanase digestion "on the blot"; then one lane was immunostained with 5D4 and the other lane with BKS-1.

\section{Immunohistochemistry and Confocal Microscopy}

Human corneal samples, fixed in $4 \%$ paraformaldehyde, were washed briefly in PBS ( $\mathrm{pH}$ 7.4), snap-frozen in tissue mountant (Cryo-Embed, Bright Instrument, Huntingdon, UK), and cryosectioned at $10 \mu \mathrm{m}$ in the midsagittal plane. Cryosections were circumscribed with a water- repellent ring and then washed briefly in PBS ( $\mathrm{pH} 7.4$ ) before immunofluorescence labeling. To generate its neoepitope site, monoclonal antibody BKS-1 required predigestion with $0.4 \mathrm{U} / \mathrm{mL}$ keratanase in 10 $\mathrm{mM}$ Tris-HCl buffer ( $\mathrm{pH} 7.4$ ) for 1 hour at $37^{\circ} \mathrm{C}$. Monoclonal antibody 5D4, which recognizes native KS chains, did not require a predigestion step. After enzymatic digestion, sections were washed in PBS containing $0.001 \%$ Tween (wash buffer and antibody diluent) for 10 minutes before blocking with normal goat serum (X0907; Dako, Carpinteria, CA) at 1:20 dilution for 30 minutes at room temperature. Adjacent tissue sections were then incubated overnight at $4^{\circ} \mathrm{C}$ with monoclonal antibodies 5D4 and BKS-1 (tissue culture supernatants) at 1:50 dilution. To check for nonspecific labeling, the primary antibody was replaced with naive mouse immunoglobulin (X0931; Dako) or an antibody diluent. After overnight incubation with primary antibody, sections were washed in buffer for 10 minutes and incubated with a goat anti-mouse FITC-conjugated secondary antibody (F0479; Dako) at 1:50 dilution for 1 hour at room temperature. Sections were washed for 10 minutes before counterstaining with $0.5 \mu \mathrm{g} / \mathrm{mL}$ propidium iodide (P-4170; Sigma) for 5 minutes at room temperature. Sections were again washed before mounting under coverslips with mountant $(\mathrm{H}-$ 1000; Vectashield; Vector Laboratories, Burlingame, CA). Representative regions of cornea, limbus, and sclera were imaged on a confocal laser scanning microscope (Sarastro 2000; GE Healthcare, Little Chalfont, Buckinghamshire, UK). Specimens were scanned using a $25-\mathrm{mW}$ argon ion laser with appropriate excitation and emission filters for simultaneous recording of fluorescein isothiocyanate (FITC; excitation maximum, $494 \mathrm{~nm}$; emission maximum, $518 \mathrm{~nm}$ ) and propidium iodide (excitation maximum, $536 \mathrm{~nm}$; emission maximum, $617 \mathrm{~nm}$ ). To reduce photobleaching, the laser output was attenuated using a $30 \%$ neutral-density filter. Specimens were examined using $\times 40$ and $\times 60$ oil immersion objectives. Series of optical sections $(512 \times 512$ pixels; approximately $0.3-\mu \mathrm{m}$ thick) were taken through the tissue section at a spacing of $0.7 \mu \mathrm{m}$ using software (Image Space; GE Healthcare) running on a Silicon Graphics (Sunnyvale, CA) workstation. Representative optical sections from each stack were presented for output as red-green overlays.

\section{Immunoelectron Microscopy}

Healthy human cornea, limbus, and sclera from three donors (age range, 24-54 years) were used to study the immunolocalization of the two different anti-KS antibodies. Small pieces of the tissue $\left(<1 \mathrm{~mm}^{3}\right)$ were fixed in freshly prepared $4 \%$ paraformaldehyde (PFA) in $0.1 \mathrm{M}$ phosphate for 2 hours at $4^{\circ} \mathrm{C}$. Tissues were processed at low temperatures and were embedded in LR White at $-20^{\circ} \mathrm{C}$ for 48 hours under ultraviolet light, as described by Akhtar et al. ${ }^{35}$

Monoclonal antibodies BKS-1 and 5D4 were used as primary antibodies to localize KS in the human and bovine corneas. The specificity of the antibody to human antigens was tested and confirmed by the supplier with immunologic assays. Antibodies were visualized with 10-nm goat anti-mouse and goat anti-mouse immunogold conjugate. The immunogold conjugates, goat serum, bovine serum, and Tween-20 were supplied by Biocell (Cardiff, UK). Monoclonal antibody BKS-1 was produced using keratanase-digested KS peptides from bovine cartilage aggrecan.

Ultrathin sections were predigested with $0.4 \mathrm{U} / \mathrm{mL}$ keratanase II in $10 \mathrm{mM}$ Tris- $\mathrm{HCl}$ buffer $\left(\mathrm{pH} \mathrm{7.4)}\right.$ for 1 hour at $37^{\circ} \mathrm{C}$ to generate a neoepitope site for monoclonal antibody BKS-1. Monoclonal antibody 5D4, which recognizes native linear pentasulfated hexasaccharide portions of KS chains, did not require a predigestion step. After enzymatic digestion, sections were immunolabeled with the primary and secondary antibodies, as described by Akhtar et al. ${ }^{35}$ Sections were stained with $2 \%$ aqueous uranyl acetate and lead citrate.

Sections were examined in a transmission electron microscope (JEOL 1010; JEOL, Tokyo, Japan), and gold particles were counted in the anterior, middle, and posterior zones of the stroma. Four images were taken from different areas from each of these regions. The center of the anterior zone was roughly within $20 \mu \mathrm{m}$ of the posterior edge of 


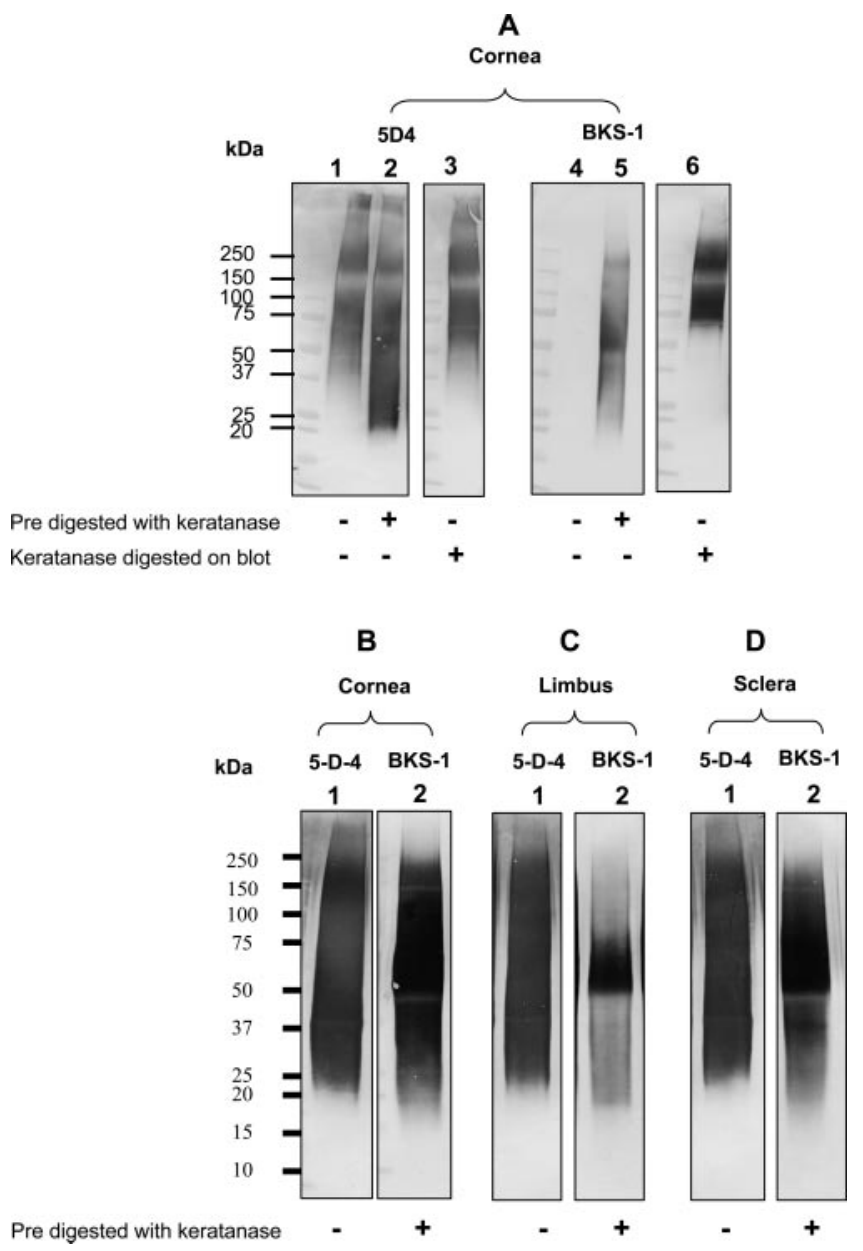

FiguRE 1. Western blot staining in normal cornea, sclera, and limbus with 5D4 and BKS-1. BKS-1 showed a less diffuse pattern of staining than 5D4 in the cornea (A), limbus (B), and sclera (C). Predigestion with keratanase before SDS-PAGE or on the blot after SDS-PAGE is indicated as $(+)$. No keratanase digestion before or after SDS-PAGE is indicated as (-).

Bowman's layer, the center of the posterior zone was roughly within $20 \mu \mathrm{m}$ of the posterior edge of Descemet's membrane, and the middle zone was estimated to be roughly in the middle of the width of the particular section under examination. This procedure was repeated three times in each sample. Approximately 30,000 gold particles were analyzed overall (AnalysIS; Soft Imaging System GmbH, Münster, Germany). Data were analyzed (SPSS, Chicago, IL) by Kolmogorov-Smirnov and Mann-Whitney $U$ tests. Graphs were drawn using the Excel program.

\section{Results}

\section{Distribution of 5D4 and BKS-1 in Normal Cornea, Limbus, and Sclera}

Without deglycosylation (-), corneal KS-PGs (e.g., lumican and keratocan) are heterogeneous and run as a smear on SDS-PAGE gels. Keratanase digestion of KS-PGs results in only partial deglycosylation (i.e., incomplete removal of the KS GAGS), resulting in the smears on SDS-PAGE. However, the amount of the smearing is reduced (e.g., Fig. $1 \mathrm{~B}(-)$ compared with Fig. $1 \mathrm{~B}(+)$. Definitive identification of lumican and keratocan core proteins would require complete deglycosylation of the KS-GAGs using keratanase, keratanase II, endo- $\beta$-galactosidase, and $\mathrm{N}$-glycanase digestion.
KS-PGs run as smears on Western blot analysis after SDSPAGE because they are heterogeneous in size given the variations in size and number of the KS chains on the PG core protein. Thus, 5D4 immunostaining detects two major smears, an upper one at approximately $200 \mathrm{kDa}$ and a lower one at approximately $75 \mathrm{kDa}$ (Fig. 1A, lane 1). Predigestion with keratanase reduces the intensity of the upper smear and causes the lower smear to move to approximately $50 \mathrm{kDa}$, with an additional smear at approximately 37 to $27 \mathrm{kDa}$ (Fig. 1A, lane 2). The smaller molecular weight smears likely result from KS-PG catabolites in the corneal extract. Keratocanase digestion on the blot (Fig. 1, lane 3), before immunolocalization with 5D4, produces essentially the same staining pattern as the undigested Western blot (Fig. 1, lane 1). This means that keratanase digestion has reduced the length of the KS chains on the KS-PGs in the corneal extract and that some of the 5D4 epitope is present on the truncated KS-GAG chains still attached to the PG core proteins. Immunostaining with BKS-1 was negative on the Western blot of native corneal KS-PGs that had not been predigested with keratanase (Fig. 1, lane 4), but strong immunostaining was detected with the KS-PGs after keratanase pretreatment (Fig. 1, lane 5). These smears now migrated at approximately 50 to $75 \mathrm{kDa}$, with some weaker staining of KSPGs at approximately $200 \mathrm{kDa}$ and even weaker reactivity with KS-PGs migrating at 27 to $37 \mathrm{kDa}$. This result indicated that the KSPGs migrating at approximately $200 \mathrm{kDa}$ and 27 to $37 \mathrm{kDa}$ had little KS containing monosulfated $\mathrm{N}$ acetyl-lactosamine disaccharides that would allow keratanase cleavage of the KS GAG chains and generation of the BKS-1 neoepitope. Immunostaining of the native corneal KSPGs after keratanase treatment on the blot (Fig. 1, lane 6) showed essentially the same staining pattern as that seen for the immunostaining with 5D4 under similar conditions (Fig. 1, lane 3). This result indicated that both species/smears of KS-PGs, found at approximately $200 \mathrm{kDa}$ and approximately $75 \mathrm{kDa}$ (Fig. 1, lanes 1, 3, 6), contained some KS that was digestible with keratanase and the production of the BKS-1 neoepitope.

Western blot analysis of corneal PG extracts showed that 5D4 staining without keratanase pretreatment (Fig. 1B, lane 1) and BKS-1 after keratanase pretreatment (Fig. 1B, lane 2) displayed a heterogeneous distribution of KS-PGs in the molecular weight range of 25 to $200 \mathrm{kDa}$. Similarly, the undigested extracts of limbus (Fig. 1C, lane 1) and sclera (Fig. 1D, lane 1) stained with $5 \mathrm{D} 4$ showed a broad spectrum of molecular mass KS-PGs from 25 to $200 \mathrm{kDa}$. In contrast, staining for BKS-1 on limbus extracts (Fig. 1C, lane 2) and sclera extracts (Fig. 1D, lane 2) after keratanase pretreatment showed a thicker, more homogeneous smear ranging from 50 to $150 \mathrm{kDa}$. Thus, BKS-1 immunostaining showed a more subtle distribution pattern than 5D4 and allowed the observation that the cornea contains more keratanase-generated KS stubs than the sclera per GAG loaded, with least the amount of these KS stubs present on KSPGs in the limbus (Figs. 1B-D).

\section{Immunohistochemistry and Confocal Microscopy}

Negative controls showed no nonspecific labeling of the corneal tissue (Fig. 2G). Comparison of immunofluorescent labeling patterns with monoclonal antibodies 5D4 and BKS-1 in the cornea, limbus, and sclera showed that both antibodies gave broadly similar distributions of KS labeling; however, the staining pattern with BKS-1 was more subtle and clearly defined (Fig. 2). In the stroma of the cornea (Figs. 2A, 2B) and the limbus (Figs. 2C, 2D), monoclonal antibodies 5D4 and BKS-1 strongly immunolocalized KS to sites between adjacent collagen fiber bundles, manifest under epifluorescence optics as highly elongated fibrillar streaks of punctuate immunolabel (Figs. 2A, 2B, insets). In the cornea, KS labeling occurred 
5D4
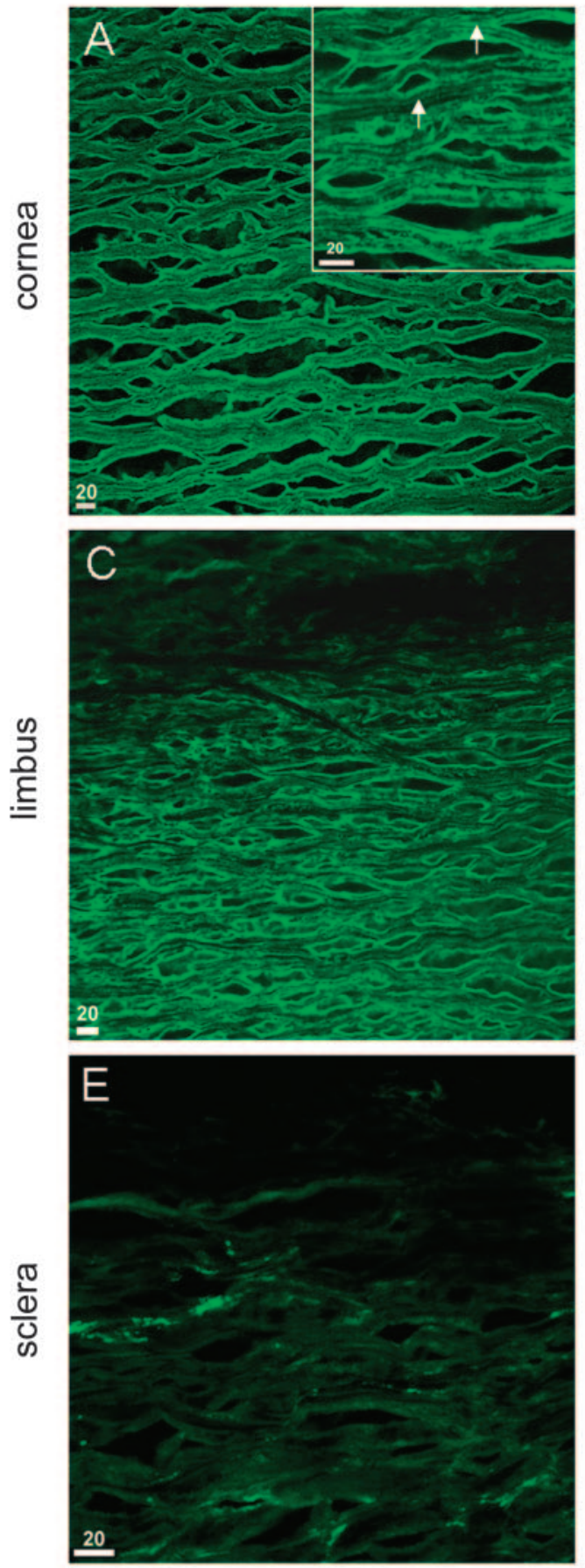

BKS-1
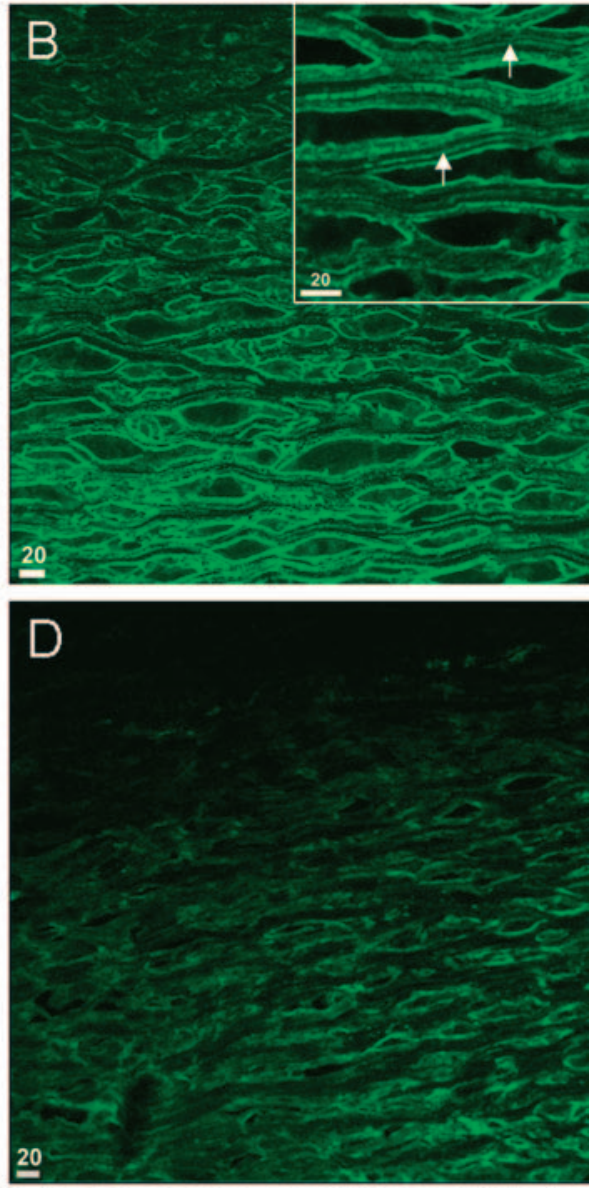

$\mathrm{F}$

FIGURE 2. Immunofluorescent localization of keratan sulfate with monoclonal antibodies 5D4 and BKS-1 in normal human cornea, limbus, and sclera. Scale bars are in iM. (A, B) In cornea, keratan sulfate is immunolocalized throughout the stroma with both KS antibodies; however, BKS-1 gives a more clearly defined staining pattern. High-power observation of KS immunostaining (inset) reveals the presence of highly elongated fibrillar streaks and rows of punctuate $\mathrm{KS}$ immunolabel interposed between adjacent collagen fiber bundles (arrows). (C, D) In limbus, both KS antibodies give strong immunofluorescent labeling of the posterior two thirds of the tissue depth. (E, F) In sclera, immunofluorescent label for KS diminishes markedly and is manifest in discrete regions between collagen fiber bundles. Note that monoclonal antibody BKS-1 gives a more restricted labeling pattern than that of 5D4. (G) Negative control.

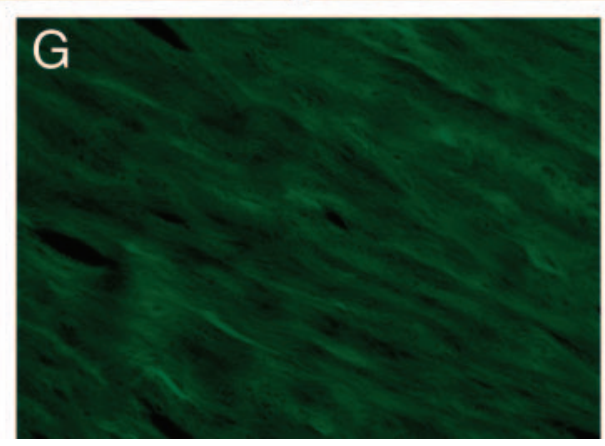



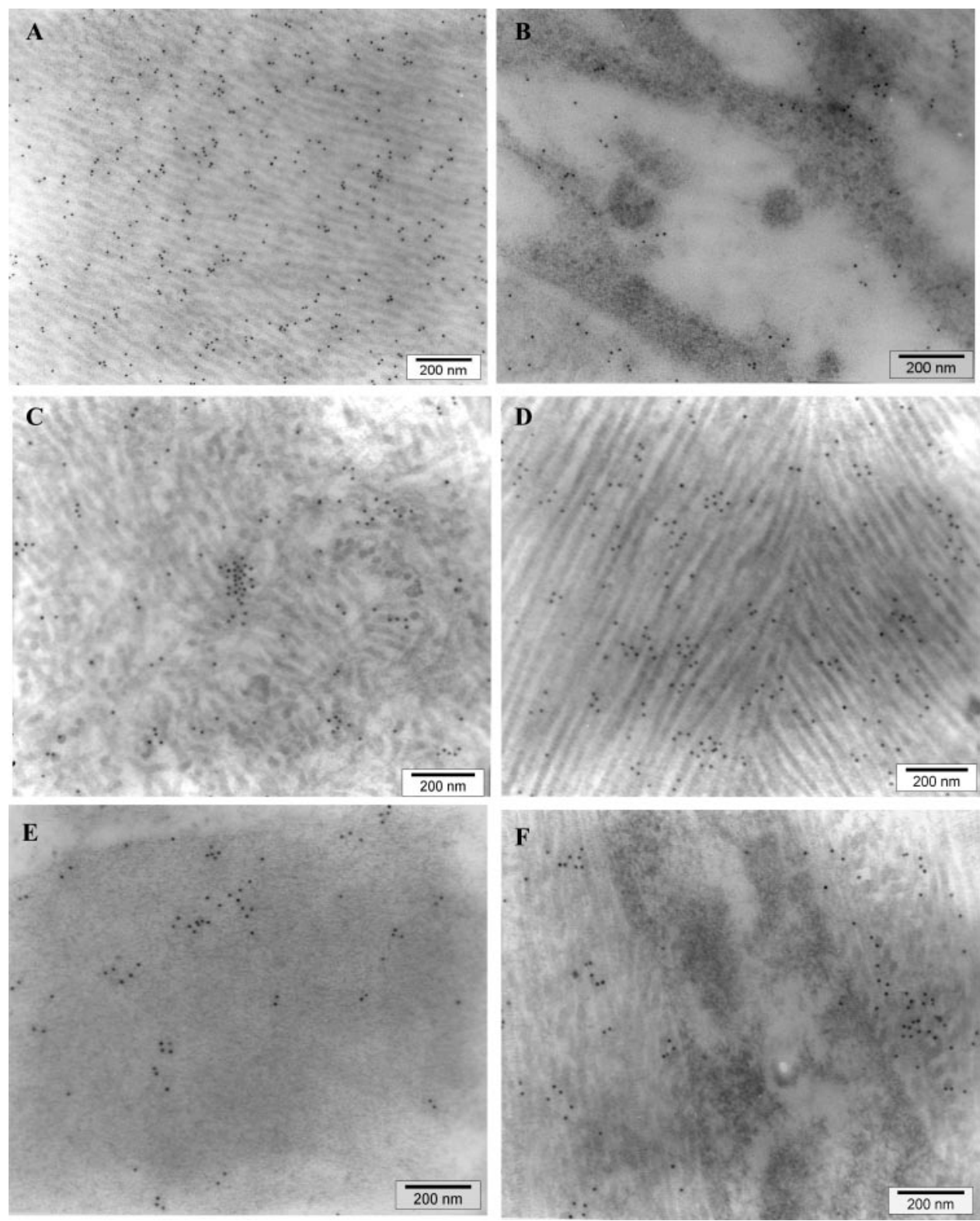

Figure 3. Immunogold localization of keratan sulfate with monoclonal antibodies 5D4 and BKS-1 in normal human cornea. (A) Strong labeling of $5 \mathrm{D} 4$ in the stroma. (B) Labeling of $5 \mathrm{D} 4$ in and around the keratocytes. Labeling of BKS-1 in (C) Bowman's layer, (D) stroma, and (E) Descemet's membrane. (F) With BKS-1, no labeling was observed in the keratocytes. throughout the depth of the tissue, but, in the limbus, KS was virtually absent from the limbal conjunctiva (upper one third of the tissue depth; Figs. 2C, 2D). In the sclera, KS immunostaining was again markedly reduced, with both antibodies showing $\mathrm{KS}$ as occurring in discrete concentrations within the tissue (Figs. 2E, 2F).

\section{Ultrastructure Distribution of BKS-1 and 5D4 in Cornea, Limbus, and Sclera}

The labeling of 5D4 was observed in the epithelium, basement membrane (not shown), throughout the stroma (Fig. 3A), and in keratocytes (Fig. 3B). There was a high density of KS-5D4 in Bowman's layer, stroma, and Descemet's membrane but moderate labeling in keratocytes. Figure 3 also shows the distribution of BKS-1 in Bowman's layer (Fig. 3C), stroma (Fig. 3D), and Descemet's membrane (Fig. 3E). Labeling of the antibody was strong in Bowman's layer, stroma, and Descemet's membrane, similar to 5D4 labeling, but no labeling was seen in the epithelium, basement membrane, keratocytes, or endothelium. A moderate quantity of the antibody was present around the keratocytes (Fig. 3F).
The labeling of 5D4 and BKS-1 was analyzed from the digital images (Figs. 4A, 4B) processed (AnalySIS; Soft Imaging System $\mathrm{GmbH}$ ). Figure 5 shows the quantitative analysis of the antibody labeling at different depths in the central cornea. There was a high density of KS-5D4 in Bowman's layer and in the anterior, middle, and posterior stroma, with no obvious differences between the regions. 5D4 labeled Descemet's membrane intensely, and the labeling was significantly higher $(P>$ 0.001) than in the other four layers (Fig. 4C). The density of KS-BKS-1 in the anterior stroma was higher than in the middle and posterior stroma, but it was similar in Bowman's layer and Descemet's layer (Fig. 4C). The intensity of 5D4 in all these regions of the cornea was significantly higher $(P>0.001)$ than of BKS-1 (Fig. 4C).

In the limbal stroma, moderate labeling of 5D4 was observed (Fig. 5A), but no labeling was seen in the limbal conjunctiva. The distribution of the antibody was uniform throughout the limbal stroma, and the labeling was less than that seen in the cornea. In the sclera, the distribution of 5D4 was uniform but very low (Fig. 5C). Labeling of BKS-1 was also observed in the limbal stroma (Fig. 5B) and sclera (Fig. 5D), but 


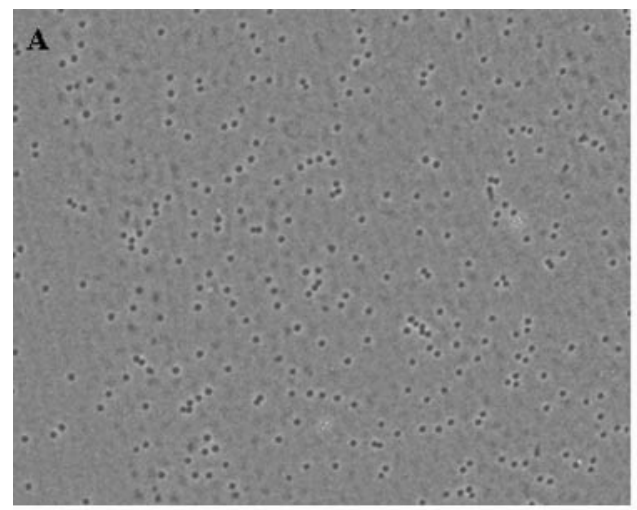

5D4 338 Ant

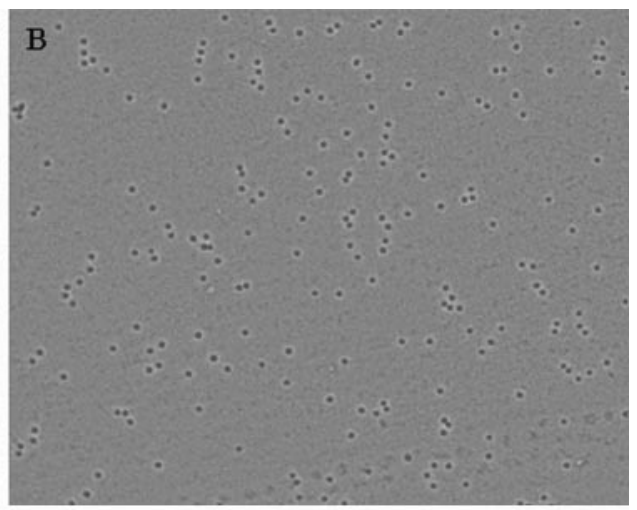

BKS-1 228 Ant
FIGURE 4. Digital images showing examples of gold particle visualization in the anterior stroma (A) using 5D4 and (B) BKS-1. (C) Graph showing the distribution of gold-particle labeling 5D4 and BKS-1 in different layers of the central normal cornea. Density of gold particles is expressed as the number of particles per $\mu \mathrm{M}^{2}$. Bars represent SEM.

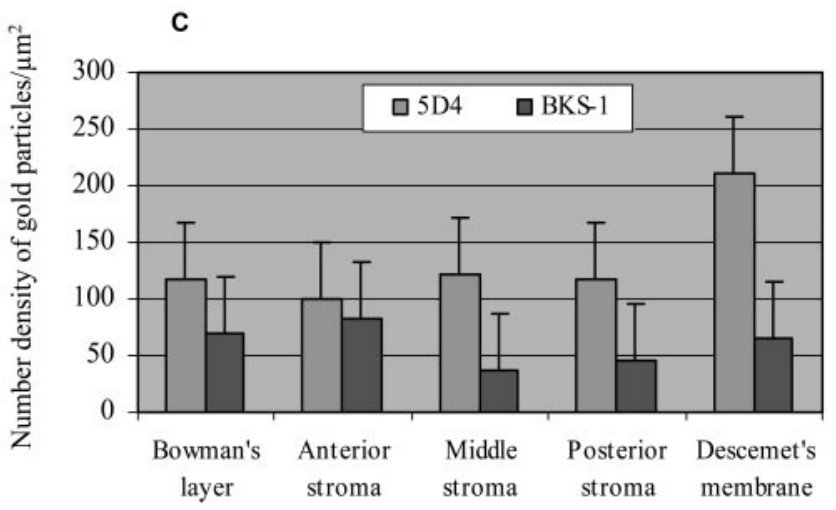

it was lower than in the cornea. The distribution of BKS-1 was not uniform; rather, the label was aggregated in places. Figure $5 \mathrm{E}$ quantifies the reduction in labeling between the limbus and the sclera using both antibodies.

\section{Discussion}

Alteration in the structure of PGs in ocular tissue has previously been assessed using antibodies against KS and dermatan sulfate. Monoclonal antibodies against KS have been useful in identifying KS during normal corneal embryonic development ${ }^{36}$ and in healing corneal wounds. ${ }^{37}$ Monoclonal antibodies against KS-PG were also used to study the structural changes in keratoconus. ${ }^{38,39}$

In the present studies both 5D4 and BSK-1 showed strong labeling in the cornea and limbus but less in the sclera. Young et al. ${ }^{40}$ showed that BKS-1 staining was lower than 5D4 staining in a single human cornea studied. We can now confirm that this is the case and that it is true for all the regions (i.e., anterior, middle, and posterior stroma) of the cornea. 5D4 potentially recognizes several linear sulfation epitopes on a single KS GAG, whereas BKS-1 only recognizes one neoepitope per KS chain if that GAG chain has been susceptible to keratanase digestion. This difference could be the reason there was more labeling of 5D4 than of BKS-1 in the different tissues examined.

Bettelheim ${ }^{41}$ investigated the glucosamine/galactosamine ratio after chromatographic separation and showed that there was a gradual increase in the ratio when proceeding from the epithelium to the endothelium of bovine cornea. The glucosamine/galactosamine ratio reflects the KS/chondroitin sulfate ratio of matrix GAGs in the cornea, and it was suggested that a gradient of KS content in the cornea increased posteri- orly. Scott and Haigh ${ }^{42}$ later reported that CS is produced in the presence of large quantities of oxygen and that KS is produced when oxygen is lacking, and it was suggested that KS stands in for CS when the oxygen supply is low. To support this hypothesis, it was shown that there was an increase in the quantity of $\mathrm{KS}$ in the stroma of corneas from various species that correlated with an increase in thickness. ${ }^{43}$ These studies were conducted with the use of Alcian blue staining, in which only sulfated groups on the KS glycosaminoglycans were stained.

In the present study, KS-specific antibody 5D4 showed only a small difference in the KS-PG (sulfate) labeling between anterior stroma and posterior stroma (not significant). On the other hand, assuming that the same proportion of antigenic sites was exposed in different sections, the differences in anterior-posterior labeling with BKS-1 implies that there were fewer KS-PG stubs in the posterior stroma than in the anterior stroma, which in turn suggests that that fewer KS-PG GAG chains were susceptible to keratanase digestion. Our results may be reconciled with those of Bettelheim ${ }^{40}$ and Scott and Haigh $^{42}$ if we hypothesize that the posterior stroma, though containing fewer BKS-1 detectible KS-GAG chains, comprises chains that are longer or more oversulfated (i.e., 5D4 positive), whereas the anterior stroma, though having more KS-PG chains, contains chains that are relatively shorter or less sulfated than those in the posterior stroma.

Monoclonal antibodies such as 5D4 recognize numerous linear pentasulfated hexasaccharide structures within one KS chain, whereas BKS-1 recognizes a single neoepitope, the 6-sulfated $\mathrm{N}$-acetyl glucosamine residue on the KS stubs after keratanase digestion. Thus, BKS-1 labeling more accurately reveals the presence of KS in the cornea, limbus, and sclera by recognizing a single epitope on KS chains. The presence of a large quantity of both antibodies reacting in the cornea suggests that 

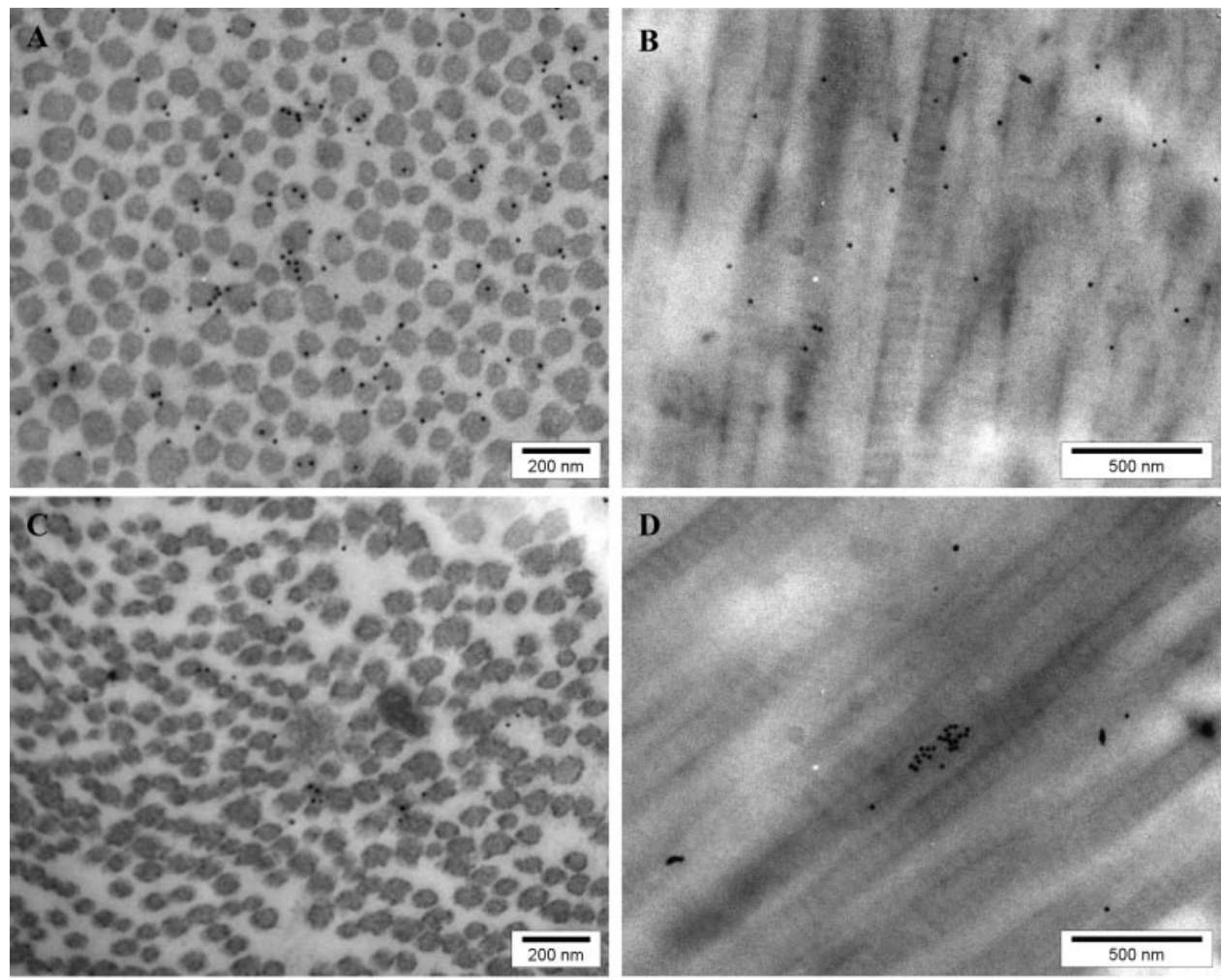

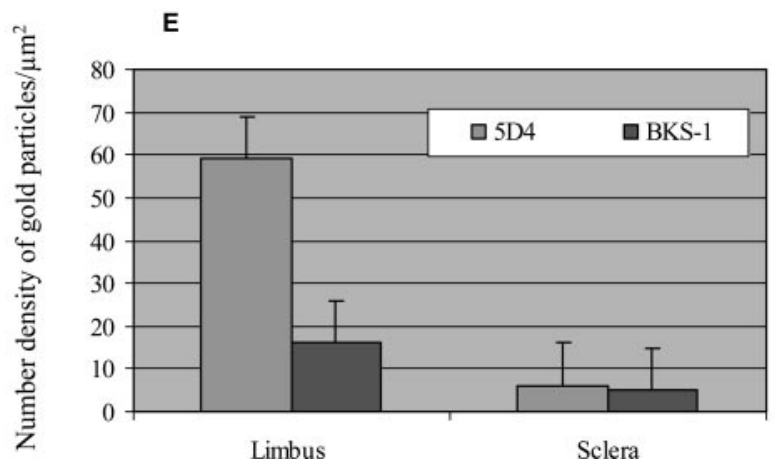

Figure 5. Immunogold localization of keratan sulfate with monoclonal antibodies 5D4 and BKS-1 in normal human limbus and sclera. Uniform distribution of 5D4 (A) in limbus and (B) in sclera. Distribution of BKS-1 (C) in limbus and (D) in sclera. (E) Graph showing distribution of goldparticle labeling 5D4 and BKS-1 in limbus and sclera. Density of gold particles is expressed as the number of particles per $\mu \mathrm{M}^{2}$. Scale bars represent SEM.

KS-PGs, as well as KS, are more prevalent in the cornea than in the limbus and sclera. The use of BKS-1 will be a helpful tool to identify a more accurate and clearly defined pattern for KS not only in the normal cornea but also in pathologic tissue such as keratoconus.

\section{References}

1. Axelson I. Heterogenecity, polydispersity and physiological role of corneal proteoglycans. Acta Ophthalmol (Copenh). 1984;62:2538 .

2. Scott JE. Morphometry of cupromeronic blue-stained proteoglycan molecules in animal corneas, versus that of purified proteoglycans stained in vitro, implies that tertiary structures contribute to corneal ultrastructure. J Anat. 1992;180:155-164.

3. Kao WW, Liu CY. Roles of lumican and keratocan on corneal transparency. Glycoconj J. 2002;19:275-285.

4. Hassell JR, Kimura JH, Hascall VC. Proteoglycan core protein families. Annu Rev Biochem. 1986;55:539-567.

5. Axelsson I, Heinegard D. Characterization of the keratan sulphate proteoglycans from bovine corneal stroma. Biochem J. 1978;169: 517-530.
6. Gregory JD, Coster L, Damle SP. Proteoglycans of rabbit corneal stroma: isolation and partial characterization. J Biol Chem. 1982; 257:6965-6970

7. Funderburgh JL, Caterson B, Conrad GW. Keratan sulfate proteoglycan during embryonic development of the chicken cornea. Dev Biol. 1986;116:267-277.

8. Hassell JR, Newsome DA, Hascall VC. Characterization and biosynthesis of proteoglycans of corneal stroma from rhesus monkey. J Biol Chem. 979;254:12346-12354.

9. Soriano ES, Campos MS, Michelacci YM. Effect of epithelial debridement on glycosaminoglycan synthesis by human corneal explants. Clin Chim Acta. 2000;295:41-62.

10. Musselmann K, Kane B, Alexandrou B, Hassell JR. Stimulation of collagen synthesis by insulin and proteoglycan accumulation by ascorbate in bovine keratocytes in vitro. Invest Ophthalmol Vis Sci. 2006; 47:5260-5266.

11. Quantock AJ, Meek KM, Ridgway AE, Bron AJ, Thonar EJ. Macular corneal dystrophy: reduction in both corneal thickness and collagen interfibrillar spacing. Curr Eye Res. 1990;9:393-398.

12. Scott JE, Haigh M. 'Small'-proteoglycan:collagen interactions: keratan sulphate proteoglycan associates with rabbit corneal collagen fibrils at the 'a' and 'c' bands. Biosci Rep. 1985;5:765774 
13. Hahn RA, Birk DE. beta-D xyloside alters dermatan sulfate proteoglycan synthesis and the organization of the developing avian corneal stroma. Development. 1992;115:383-393.

14. Hassell JR, Newsome DA, Krachmer JH, Rodrigues MM. Macular corneal dystrophy: failure to synthesize a mature keratan sulfate proteoglycan. Proc Natl Acad Sci U S A. 1980;77:3705-3709.

15. Funderburgh JL, Funderburgh ML, Mann MM, Conrad GW. Physical and biological properties of keratan sulphate proteoglycan. Biochem Soc Trans. 1991;19:871-876.

16. Blochberger TC, Vergnes JP, Hempel J, Hassell JR. cDNA to chick lumican (corneal keratan sulfate proteoglycan) reveals homology to the small interstitial proteoglycan gene family and expression in muscle and intestine. J Biol Chem. 1992;267:347-352.

17. Funderburgh JL, Funderburgh ML, Brown SJ, et al. Sequence and structural implications of a bovine corneal keratan sulfate proteoglycan core protein: protein $37 \mathrm{~B}$ represents bovine lumican and proteins 37A and 25 are unique. J Biol Chem. 1993;268:1187411880 .

18. Corpuz LM, Funderburgh JL, Funderburgh ML, Bottomley GS, Prakash S, Conrad GW. Molecular cloning and tissue distribution of keratocan: bovine corneal keratan sulfate proteoglycan 37A. J Biol Chem. 1996;271:9759-9763.

19. Funderburgh JL, Mitschler RR, Funderburgh ML, Roth MR, Chapes SK, Conrad GW. Macrophage receptors for lumican: a corneal keratan sulfate proteoglycan. Invest Ophthalmol Vis Sci. 1997;38: $1159-1167$.

20. Ying S, Shiraishi A, Kao CW, et al. Characterization and expression of the mouse lumican gene. J Biol Chem. 1997;272:30306-30313.

21. Liu CY, Birk DE, Hassell JR, Kane B, Kao WW. Keratocan-deficient mice display alterations in corneal structure. J Biol Chem. 2003; 278:21672-21677.

22. Meyer K, Linker A, Davidson EA, Weissman B. The mucopolysaccharides of the cornea. J Biol Chem. 1953;205:611-616.

23. Hascall VC. Structure and biosynthesis of proteoglycans with keratan sulfate. Prog Clin Biol Res. 1982;110:3-15.

24. Nieduszynski IA, Huckerby TN, Dickenson JM, Brown GM, Tai GH, Bayliss MT. Structural aspects of skeletal keratan sulphates. Biochem Soc Trans. 1990;18:792-793.

25. Greiling H. Structure and biological functions of keratan sulfate proteoglycans. EXS. 1994;70:101-122

26. Fischer DC, Haubeck HD, Eich K, et al. A novel keratan sulphate domain preferentially expressed on the large aggregating proteoglycan from human articular cartilage is recognized by the monoclonal antibody 3D12/H7. Biochem J. 1996;318:1051-1056.

27. Krusius T, Finne J, Margolis RK, Margolis RU. Identification of an O-glycosidic mannose-linked sialylated tetrasaccharide and keratan sulfate oligosaccharides in the chondroitin sulfate proteoglycan of brain. J Biol Chem. 1986;261:8237-8242.

28. Caterson B, Christner JE, Baker JR. Identification of a monoclonal antibody that specifically recognizes corneal and skeletal keratan sulfate: monoclonal antibodies to cartilage proteoglycan. $J$ Biol Chem. 1983;258:8848-8854.
29. Scudder P, Tang PW, Hounsell EF, Lawson AM, Mehmet H, Feizi T. Isolation and characterization of sulphated oligosaccharides released from bovine corneal keratan sulphate by the action of endo-beta-galactosidase. Eur J Biochem. 1986;157:365-373.

30. Hounsell EF, Feeney J, Scudder P, Tang PW, Feizi T. 1H-NMR studies at $500 \mathrm{MHz}$ of a neutral disaccharide and sulphated di-, tetra-, hexa- and larger oligosaccharides obtained by endo-betagalactosidase treatment of keratan sulphate. Eur J Biochem. 1986; 157:375-384

31. Mehmet H, Scudder P, Tang PW, Hounsell EF, Caterson B, Feizi T. The antigenic determinants recognized by three monoclonal antibodies to keratan sulphate involve sulphated hepta- or larger oligosaccharides of the poly(N-acetyllactosamine) series. Eur J Biochem. 1986;157:385-391.

32. Tang PW, Scudder P, Mehmet H, Hounsell EF, Feizi T. Sulphate groups are involved in the antigenicity of keratan sulphate and mask in antigen expression on their poly-N-acetyllactosamine backbones: an immunochemical and chromatographic study of keratan sulphate oligosaccharides after desulphation or nitrosation. Eur J Biochem. 1986;160:537-545.

33. Kerr BC. Keratan Sulphate Metabolism in Connective Tissue Proteoglycans. Cardiff: Cardiff University; 2005. PhD thesis.

34. Heinegard D, Axelsson I. Distribution of keratan sulfate in cartilage proteoglycans. J Biol Chem. 1977;25:1971-1979.

35. Akhtar S, Bron AJ, Hawksworth NR, Bonshek RE, Meek KM. Ultrastructural morphology and expression of proteoglycans, betaig-h3, tenascin-C, fibrillin-1, and fibronectin in bullous keratopathy. $\mathrm{BrJ}$ Ophthalmol. 2001;85:720-731.

36. Funderburgh JL, Caterson B, Conrad GW. Distribution of proteoglycans antigenically related to corneal keratan sulfate proteoglycan. J Biol Chem. 1987;262:11634-11640.

37. Funderburgh JL, Cintron C, Covington HI, Conrad GW. Immunoanalysis of keratan sulfate proteoglycan from corneal scars. Invest Ophthalmol Vis Sci. 1988;29:1116-1124.

38. Funderburgh JL, Funderburgh ML, Rodrigues MM, Krachmer JH, Conrad GW. Altered antigenicity of keratan sulfate proteoglycan in selected corneal diseases. Invest Ophthalmol Vis Sci. 1990;31: $419-428$.

39. Sawaguchi S, Yue BY, Chang I, Sugar J, Robin J. Proteoglycan molecules in keratoconus corneas. Invest Ophthalmol Vis Sci. 1991;32:1846-1853

40. Young RD, Akama TO, Liskova P, et al. Differential immunogold localisation of sulphated and unsulphated keratan sulphate proteoglycans in normal and macular dystrophy cornea using sulphation motif-specific antibodies. Histochem Cell Biol. 2007;127:115-120.

41. Bettelheim FA, Goetz D. Distribution of hexosamines in bovine cornea. Invest Ophthalmol. 1976;15:301-304.

42. Scott JE, Haigh M. Keratan sulphate and the ultrastructure of the cornea and cartilage: a "stand-in" for chondroitin sulphate in conditions of oxygen lack? J Anat. 158:95-108.

43. Scott JE, Bosworth TR. The comparative chemical morphology of the mammalian cornea. Basic Appl Histochem. 1990;34:35- 42. 